\title{
Изотипные гетероструктуры $n$-AIGaAs/n-GaAs, оптимизированные для эффективной межзонной излучательной рекомбинации при накачке электрическим током
}

\author{
(C) О.С. Соболева, С.О. Слипченко, Н.А. Пихтин \\ Физико-технический институт им. А.Ф. Иоффе Российской академии наук, \\ 194021 Санкт-Петербург, Россия \\ E-mail: soboleva@mail.ioffe.ru
}

Поступила в Редакцию 11 января 2021 г.

В окончательной редакции 18 января 2021 г.

Принята к публикации 18 января 2021 г.

Проведен анализ транспорта носителей заряда при накачке током изотипной AlGaAs/GaAs-гетероструктуры, оптимизированной для эффективной межзонной излучательной рекомбинации. Для анализа особенностей транспорта носителей заряда использовались модели: дрейф-диффузионная, дрейф-диффузионная с полевой зависимостью подвижности, а также энергетического баланса. Показано, что при низких токах уровень излучательной рекомбинации в активной области выше в модели энергетического баланса из-за более эффективного накопления носителей, генерируемых посредством ударной ионизации. Это объясняется наличием на гетерогранице эффекта „velocity overshoot“, возникающего из-за резкого изменения концентрации электронов и электрического поля на гетеропереходе, тогда как в дрейф-диффузионном приближении скорость дрейфа в данной области равна насыщенной, и наблюдается снижение потенциального барьера из-за накопления большой концентрации электронов вблизи гетероперехода. При токах $>40$ А уровень излучательной рекомбинации в активной области выше в дрейф-диффузионнном приближении, что объясняется более высокими значениями напряженности поля и темпа ударной ионизации в слое с доменом электрического поля. Продемонстрировано увеличение тока излучательной рекомбинации в активной области более чем на $50 \%$ до 13.5 А (при токе накачки $100 \mathrm{~A}$ ) и максимальной внутренней квантовой эффективности до 16\% (при токе 40 А) при уменьшении толщины слоя накопления неравновесных носителей заряда до 100 нм.

Ключевые слова: гетероструктуры, изотипные структуры, ударная ионизация, модель энергетического баланса, излучательная рекомбинация.

DOI: $10.21883 /$ FTP.2021.05.50831.9598

\section{1. Введение}

Первые работы, посвященные исследованию особенностей процессов инжекции в изотипных структурах, были проведены более 40 лет назад для структур на основе кремния $[1,2]$. Было показано, что структуры типа $n^{+}$(сильно легированный слой) $/ n^{0}$ (слабо легированный слой $) / n^{+}$(сильно легированный слой) характеризуются заметной нелинейностью вольт-амперной характеристики (BAX), которая связана с эффектом насыщения скорости дрейфа основных носителей заряда в области сильного электрического поля и включением процесса ударной ионизации [3]. Проведенные позже экспериментальные и теоретические исследования процессов транспорта в изотипных гетероструктурах показали, что, кроме нелинейности ВАХ, также наблюдается излучательная рекомбинация, связанная с генерацией неосновных носителей заряда за счет ударной ионизации в домене сильного электрического поля [4,5]. В работе [5] рассматривались изотипные гетероструктуры, где с помощью спонтанного излучения в квантово-размерной области визуализировалась токовая динамика формирования областей с сильными электрическими полями и ударной ионизации в них, при комнатной температуре. Однако данная работа была сосредоточена на исследова- нии транспорта носителей, и рассматриваемая структура не была оптимальной для накопления высоких концентраций неравновесных носителей и, соответственно, для получения высокой эффективности излучательной рекомбинации. Таким образом во всех случаях вклад излучательной рекомбинации был несущественным и анализ структур с точки зрения возможности получения эффективной излучательной рекомбинации не проводился.

Вместе с этим использование изотипных гетероструктур для получения излучательной рекомбинации может стать решением ряда существующих в настоящее время проблем. В частности, не всегда может быть реализована диодная $p-n$-структура, обеспечивающая эффективную инжекцию электронов и дырок в активную область. Например, существуют технологические трудности при создании полупроводниковых слоев с $p$-типом проводимости в материалах $\mathrm{HgCdTe}$ или $\mathrm{AlGaN} \mathrm{[6-9].} \mathrm{Как} \mathrm{аль-}$ тернатива прямой токовой накачке может использоваться оптическая накачка или накачка электронным лучом, что не всегда эффективно или же может значительно усложнять систему.

Существует и другой подход, в котором используется изотипная гетероструктура, а носители противоположного знака генерируются посредством ударной иони- 
Основные параметры модели для слоев с различным составом слоев $\left(\mathrm{Al}_{x} \mathrm{Ga}_{1-x} \mathrm{As}\right)$

\begin{tabular}{c|c|c|c|c|c|c|c|c|c}
\hline$x$ & $\tau_{n, \text { rel }, \text { пс }}$ & $A_{n}$, отн. ед. & $B_{n}$, отн. ед. & $\beta_{n}$, отн. ед. & $A_{p}$, отн. ед. & $B_{p}$, отн.ед. & $\beta_{p}$, отн. ед. & $\mu_{n 0}, \mathrm{~cm}^{2} / \mathrm{Bc}^{5}$ & $\mu_{p 0}, \mathrm{~cm}^{2} / \mathrm{Bc}$ \\
\hline 0 & 0.5 & $1.9 \cdot 10^{5}$ & $5.75 \cdot 10^{5}$ & 1.82 & $2.22 \cdot 10^{5}$ & $6.57 \cdot 10^{5}$ & 1.75 & 6500 & 300 \\
0.3 & 0.32 & $2.21 \cdot 10^{5}$ & $7.64 \cdot 10^{5}$ & 2.0 & $2.791 \cdot 10^{5}$ & $8.47 \cdot 10^{5}$ & 1.9 & 1200 & 110
\end{tabular}

зации. Преимуществами такого подхода являются не только возможность реализации электрической инжекционной накачки в системах материалов, где сложно создать одновременно слои $p$ - и $n$-типов проводимости, но и расширение типов возможных конструкций полупроводниковых светоизлучающих приборов и способов их оптимизации. К примеру, в гетероструктурах лазерных диодов слои с высоким легированием p-типа вносят высокие внутренние оптические потери за счет более высокого значения сечения поглощения на свободных носителях для дырок [10-12].

Однако присутствие ударной ионизации часто трактуется как негативный фактор, который может приводить к разрушению прибора. Тем не менее на сегодняшний день существует множество примеров приборов, в которых управляемая ударная ионизация используется для генерации неравновесных носителей заряда, в основном это структуры транзисторного и тиристорного типа [13-16].

Цель данной работы - сравнительный анализ результатов численного моделирования процессов транспорта в изотипной гетероструктуре $\mathrm{AlGaAs} / \mathrm{GaAs}$, разработанной для эффективной излучательной рекомбинации. Для сравнительного анализа использовано три модели: дрейф-диффузионная (DD: drift diffusion - дрейфдиффузионная), дрейф-диффузионная с полевой зависимостью подвижности (DDFDM: drift diffusion field dependence mobility - дрейф-диффузионная с полевой зависимостью подвижности), а также энергетического баланса с полевой зависимостью подвижности (EBFDM: energy balance field dependence mobility - энергетического баланса с полевой зависимостью подвижности). Выбор системы материалов обусловлен высоким развитием технологии полупроводниковых гетероструктур $\mathrm{AlGaAs} / \mathrm{GaAs}$, которые могут быть реализованы с высоким оптическим и кристаллическим качеством и возможностью создавать приборы, выдерживающие плотности тока накачки до 100 кА/ $\mathrm{cm}^{2}[11,14]$. Также данная система материалов хорошо изучена, что делает подбор параметров модели более простым и надежным. Рассматривался только $n$-тип проводимости из-за более высокой подвижности электронов.

\section{2. Описание модели}

В настоящее время существует достаточно большое количество моделей, описывающих транспорт носителей заряда в многослойных полупроводниковых гетероструктурах. В большинстве случаев для описания транспорта носителей заряда в таких структурах используется дрейф-диффузионная (DD: drift diffusion дрейф-диффузионная) модель [17]. Ее выбор обусловлен простотой описания транспорта носителе, а также доступностью верифицированных параметров модели для широкого спектра материалов.

Основной особенностью исследуемых в данной работе структур являются эффекты ударной ионизации и транспорта носителей заряда в сильных электрических полях. Данные эффекты в DDFDM-модели описываются с помощью полевых зависимостей подвижностей носителей заряда $\mu_{n, p}(x)$ - и коэффициентов ударной ионизации, $\alpha_{n, p}(x)-$ в точке $x$ и определяются напряженностью поля $E(x)$ в этой точке:

$$
\begin{gathered}
\mu_{n, p}(x)=\mu_{0_{n, p}}\left(1+\left(\frac{\mu_{0_{n, p}} E(x)}{v_{\text {sat }_{n, p}}}\right)^{\beta_{n, p}}\right)^{-\frac{1}{\beta_{n, p}}}, \\
\alpha_{n, p}(x)=A_{n, p} \exp \left(-\frac{B_{n, p}}{E(x)}\right),
\end{gathered}
$$

где $\mu_{0_{n, p}}, v_{\mathrm{sat}_{n, p}}, \beta_{n, p}, A_{n, p}, B_{n, p}-$ параметры модели, значения которых приведены в таблице. В случаe DD-модели без полевой зависимости подвижности $\mu_{n, p}(x)=$ const $=\mu_{0_{n, p}}$.

Несмотря на то что в данной работе рассматривается структура с толщинами слоев много больше длины энергетической релаксации электрона, при движении электронов в сильном электрическом поле, когда градиент поля не удовлетворяет соотношению $d E / d x \ll \Delta E / l_{\text {rel }}$ (что реализуется в нашем случае вблизи гетерограниц и переходов $\left.n_{0} / n_{+}\right)$, носители могут не успевать отдавать накапливаемую энергию решетке, т.е. может происходить разогрев носителей заряда $[10,18]$. В таком случае происходит запаздывание между значением напряженности электрического поля и средней энергией электронов, зависящей от температуры $T_{n}$, которое характеризуется временем релаксации энергии $\left(\tau_{\text {rel }}\right)$. Таким образом, даже при рассмотрении гетероструктур с микронными толщинами могут проявляться эффекты разогрева носителей. В данной работе мы использовали модель энергетического баланса с учетом полевой зависимости подвижности (EBFDM) как наиболее простой способ учета разогрева носителей $[19,20]$. При этом в EBFDMприближении рассматривался только транспорт электронов, а для описания транспорта дырок, как более тяжелых носителей, во всех случаях использовалось более простая DDFDM-модель. В данном приближении подвижность электронов зависит не напрямую от напряженности поля в конкретной точке $E(x)$ (см. (1) 
и (2)), а от эффективного электрического поля $E_{\text {eff }}(x)$, зависящего от температуры электронов $T_{n}$ :

$$
\begin{gathered}
\mu_{n}\left(E_{\mathrm{eff}}\right) E_{\mathrm{eff}}^{2}=\frac{3 k_{\mathrm{B}}\left(T_{n}-T_{L}\right)}{2 q \tau_{n, \mathrm{rel}} v_{\mathrm{sat}, n}} \\
\mu_{n}\left(E_{\mathrm{eff}}=\mu_{n 0}\left(1+\left(\frac{\mu_{n 0} E_{\mathrm{eff}, n}}{v_{\mathrm{sat}, n}}\right)^{\beta_{n}}\right)^{-\frac{1}{\beta_{n}}},\right.
\end{gathered}
$$

где $T_{L}$ - температура решетки; $\tau_{n, \text { rel }}-$ энергетическое время релаксации электронов; $q$ - элементарный заряд; $k_{\mathrm{B}}$ - постоянная Больцмана. Значение $E(x)$, используемое в выражении для коэффициента ударной ионизации, для модели $\mathrm{EBFDM}$ заменяется на $E_{\text {eff }}(x)$ :

$$
\alpha_{n}\left(E_{\mathrm{eff}}\right)=A_{n} \exp \left(-\frac{B_{n}}{E_{\mathrm{eff}}}\right) .
$$

В используемых моделях не учитывалась отрицательная дифференциальная подвижность (ОДП) в GaAs, так как, во-первых, влияние ОДП более выражено в нестационарных задачах, а, во-вторых, пренебрежение ОДП существенно улучшает сходимость вычислительного процесса и экономит вычислительные ресурсы. В данной задаче предполагался импульсный режим накачки структуры с длительностью $\sim 100$ нс, когда за время импульса система успевает прийти в равновесное состояние, но не успевает существенно нагреться. Таким образом, в таком подходе можно решать стационарную задачу без разогрева кристаллической решетки. Поставленные задачи решались с помощью пакета Silvaco Atlas.

Для оценки эффективности накопления неравновесной концентрации в InGaAs квантовой яме (КЯ) использовался ток излучательной рекомбинации $\left(I_{R A D}\right)$ :

$$
I_{R A D}=R_{R A D} \cdot V_{Q W} \cdot q,
$$

где $R_{R A D}-$ скорость излучательной рекомбинации, $V_{Q W}$ - объем КЯ. В активной области учитывалась излучательная рекомбинация и рекомбинация ШоклиРида-Холла (ШРХ), время жизни ШРХ в КЯ равнялось 3 нс, коэффициент $B=2 \cdot 10^{-10} \mathrm{~cm}^{3} \cdot \mathrm{c}^{-1}$. В объемном материале время жизни ШРХ равнялось 10 нс, коэффициент $B=2 \cdot 10^{-10} \mathrm{~cm}^{3} \cdot \mathrm{c}^{-1}$. КЯ на основе InGaAs рассчитывалась без учета напряжения, ширина запрещенной зоны 1.04 эВ. Коэффициент $\mu_{0_{n, p}}$ в (1), (4) в $\mathrm{AlGaAs}$ высоколегированных слоях оставался неизменным и равнялся 800 и $70 \mathrm{~cm}^{2} / \mathrm{B} \cdot \mathrm{c}$ для электронов и дырок соответственно. Время энергетической релаксации выбиралось на основе теоретических и экспериментальных данных, приведенных в [21-24], и приведено в таблице.

\section{3. Исследуемая структура}

Проведенные ранее исследования изотипных полупроводниковых гетероструктур показали, что эффективным источником неосновных носителей заряда может

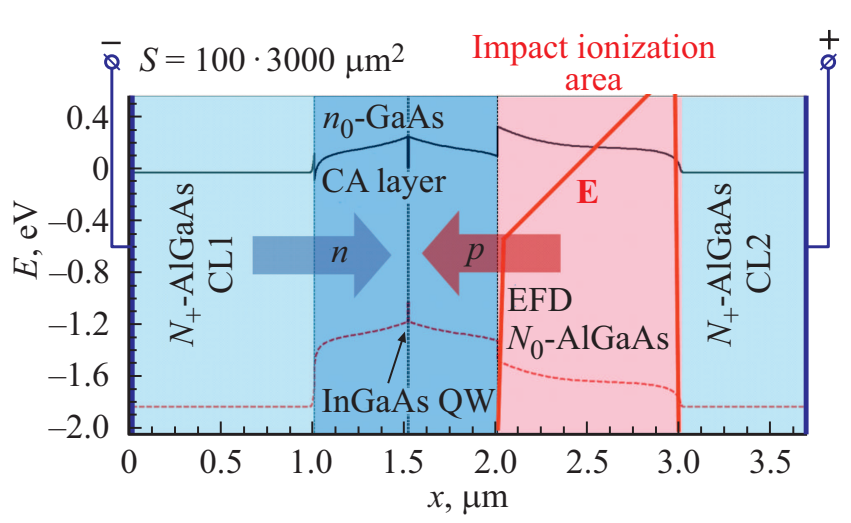

Рис. 1. Схематическое изображение исследуемой изотипной гетероструктуры.

служить домен сильного электрического поля за счет активной ударной ионизации $[5,14,15,25]$. На основании этого основные принципы формирования конструкции изотипной гетероструктуры для эффективной излучательной рекомбинации можно сформулировать следующим образом: (1) конструкция гетероструктуры должна включать часть, отвечающую за ударную ионизацию; (2) конструкция гетероструктуры должна содержать область, обеспечивающую эффективный сбор носителей заряда; (3) конструкция гетероструктуры должна предусматривать решения, подавляющие утечку носителей заряда из области накопления носителей заряда.

Проведенные ранее исследования показали, что активная ударная ионизация может происходить в области изотипного гетероперехода при смещении в обратном направлении и уровне легирования $10^{15}-10^{16} \mathrm{~cm}^{-3}[5,14]$. Накопление носителей в нашем случае может быть реализовано за счет объемного узкозонного слоя, расположенного вне области ударной ионизации. При этом для повышения эффективности сбора в данном узкозонном слое может быть использована квантово-размерная активная область. Подавление утечки неосновных носителей может быть реализовано за счет использования широкозонных эмиттеров на границе с узкозонным слоем. Используя указанные принципы, была выбрана структура, представленная схематически на рис. 1.

Базовая гетероструктура состояла из двух $N_{+}$высоколегированных эмиттеров: CL1 и CL2 (CL - Cladding Layer) (рис. 1) (AlGaAs (30\%), $\left.N_{D}=10^{18} \mathrm{~cm}^{-3}\right)$, между которыми был расположен слой накопления неравновесных носителей заряда (CA - carrier accumulation) и слой, в котором формировался домен электрического поля (EFD - electric field domain). Слой СА образован за счет узкозонного GaAs толщиной 1 мкм и $\mathrm{InGaAs}$ квантовой ямы толщиной 10 нм, расположенной в центре этого слоя $\left(N_{D}=10^{15} \mathrm{~cm}^{-3}\right)$. Слой EFD сформирован широкозонным $\mathrm{AlGaAs}(30 \%)$ толщиной 1 мкм $\left(N_{D}=10^{15} \mathrm{~cm}^{-3}\right)$. Полярность внешнего напряжения по- 
казана на рис. 1. Площадь исследуемой гетероструктуры равнялась $3 \cdot 10^{-3} \mathrm{~cm}^{2}$.

\section{4. Анализ результатов}

Рассмотрим основные принципы работы исследуемой структуры (рис. 1). В равновесии, без внешнего смещения, в области гетероперехода за счет некомпенсированного заряда (избыточные электроны со стороны СА и объемный положительный заряд со стороны EFD) формируется локальное электрическое поле. При внешнем смещении с полярностью, указанной на рис. 1, наблюдается увеличение напряженности электрического поля на границе низколегированных слоев CA/EFD. Кроме этого, увеличение внешнего напряжения сопровождается ростом тока и расширением домена электрического поля в сторону границы EFD/CL2, так как со стороны узкозонного слоя СА накапливается более высокая концентрация свободных носителей заряда, которая сдерживает расширение электрического поля. Когда плотность тока в структуре становится сравнимой с насыщенной $J_{\text {sat }}$ (в данном случае $J_{\text {sat }}=q v_{\text {sat }} N_{D} \sim 0.96 \mathrm{kA} / \mathrm{cm}^{2} \sim 2.9$ A, где $v_{\text {sat }}-$ насыщенная скорость электронов в EFD-слое), градиент поля меняет свой знак и начинает образовываться анодный домен на границе EFD/CL2. Более подробно процесс образования домена электрического поля в подобных структурах описан в $[5,14]$. Рост напряженности электрического поля приводит к увеличению темпа ударной ионизации в EFD-слое. Это формирует поток дырок из EFD- в CA-слой и обеспечивает их накопление в InGaAs активной области, и, следовательно, ведет к увеличению скорости излучательной рекомбинации.

На рис. 2 показаны зависимости тока излучательной рекомбинации в КЯ $\left(I_{R A D}\right)$ от тока, который протекает через структуру $(I)$. Расчеты данных зависимостей проведены в моделях DD, DDFDM, EBFDM. Видно, что в DD-модели, в которой отсутствует насыщение скорости дрейфа, ток излучательной рекомбинации практически отсутствует. Это связано с высокой подвижностью носителей заряда в сильных электрических полях. Как следствие, эффект накопления носителей заряда в слое EFD практически отсутствует и формирования домена с высокой напряженностью электрического поля не происходит (рис. 3). Данный результат представляется физически некорректным (так как дрейфовая скорость не ограничена в сильных электрических полях, присутствующих в структуре - см. рис. 3 кривая 3), поэтому далее он подробно рассматриваться не будет. Однако отсюда следует, что насыщение скорости дрейфа играет существенную роль в работе рассматриваемой структуры. Электроны, инжектированные из слоя СА в широкозонный EFD-слой, попадают в область с сильным электрическим полем, в результате их дрейфовая скорость падает до насыщенной, что приводит к накоплению избыточного заряда в области сильного поля, что

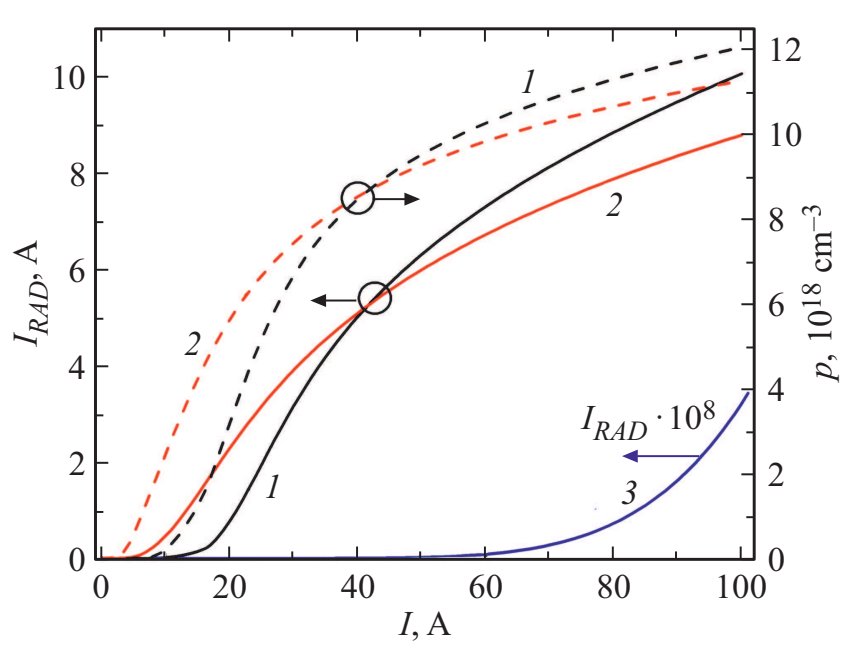

Рис. 2. Зависимость тока излучательной рекомбинации $\left(I_{R A D}\right)$ (сплошные линии) и концентрации дырок $(p)$ в InGaAs активной области (пунктир) от тока в различных моделях: 1 DD FDM, 2 - EB FDM, $3-$ DD.

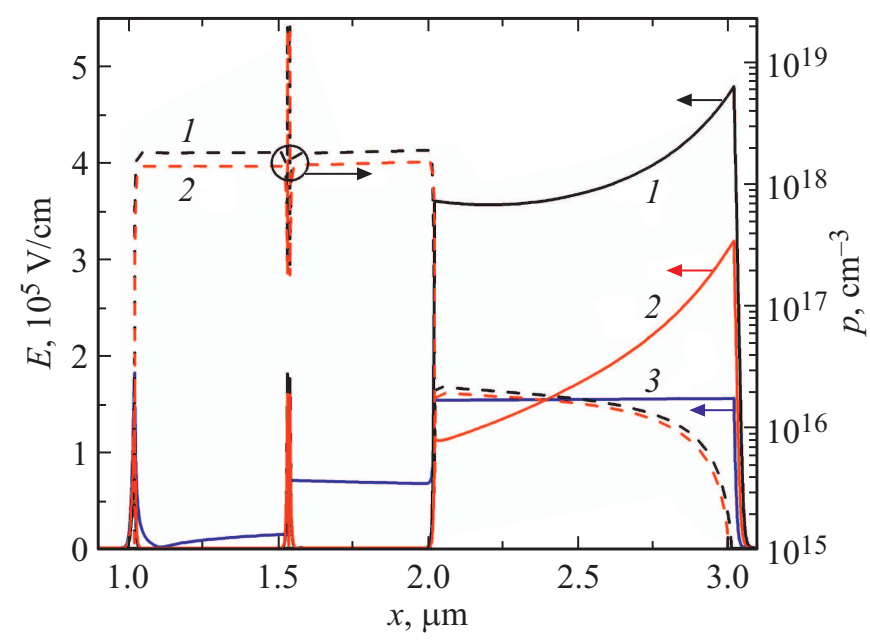

Рис. 3. Распределения напряженности электрического поля (сплошные линии) и концентрации дырок (пунктир) в исследуемой гетероструктуре при токе $100 \mathrm{~A}$ в различных моделях: 1 - DDFDM, 2 - EBFDM, 3 - DD.

в свою очередь приводит к росту напряженности поля в данном слое и изменению его градиента.

При рассмотрении транспорта в рамках модели DDFDM, учитывающей насыщение скорости дрейфа в сильных электрических полях, получено максимальное значение тока излучательной рекомбинации $10 \mathrm{~A}$ при полном токе через структуру $100 \mathrm{~A}$ (рис. 2). Данный факт объясняется тем, что расчеты в DDFDM дают большее значение напряженности электрического поля в EFD-слое, что связано с более низкой скоростью дрейфа вблизи гетеропереходов. Несмотря на микронную толщину данного слоя, поле в структуре меняется резко, при этом в DDFDM-модели скорость дрейфа напрямую зависит от напряженности поля и падает до насыщенной, 


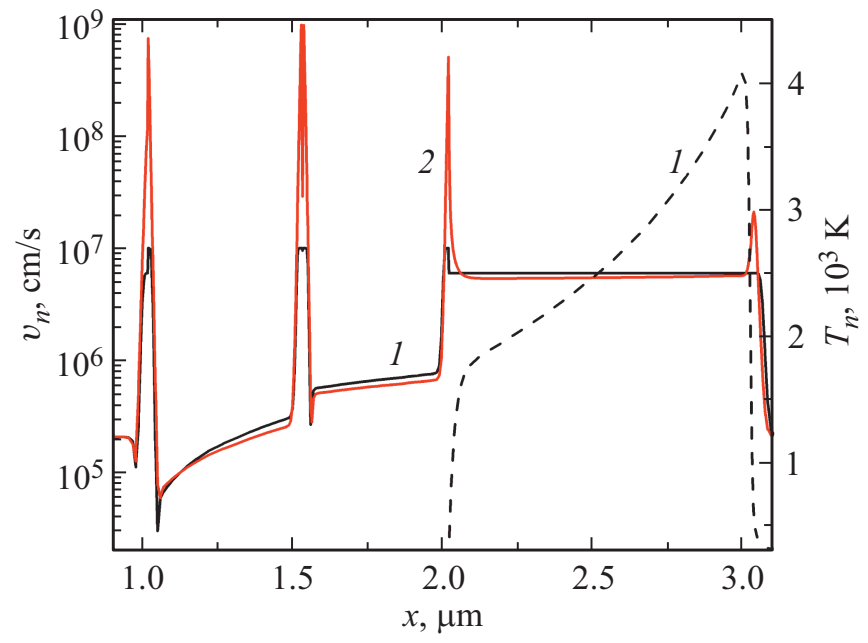

Рис. 4. Распределение скорости дрейфа (сплошные линии) и температуры электронов в области EFD-слоя (пунктир) в исследуемой гетероструктуре при токе $100 \mathrm{~A}$ в различных моделях: 1 - DD FDM, 2 - EB FDM.

как только носители попадают в область сильного электрического поля. В EBFDM-модели наблюдается эффект „velocity overshoot“ [19,23,24], а насыщенная скорость зависит от температуры электронов (рис. 4), поэтому в данном приближении необходима меньшая концентрация носителей для обеспечения транспорта и напряженность поля, в частности на гетерогранице, ниже. Кроме того, из-за отличий в описании процесса ударной ионизации в DDFDM-модели при больших токах может наблюдаться переоценка темпа ударной ионизации, из-за того, что коэффициенты ударной ионизации напрямую зависят от напряженности поля $[18,23]$.

Стоит отметить, что и в DDFDM-, и в EBFDMмодели при токах $>10 \mathrm{~A}$ в области InGaAs активной области концентрация неравновесных дырок превышает $3 \cdot 10^{18} \mathrm{~cm}^{-3}$ (рис. 3), что делает возможным создание униполярных приборов с высоким материальным усилением оптического излучения.

Интересен тот факт, что при токах < 40 A EBFDMмодель дает больший ток излучательной рекомбинации по сравнению с DDEFD-моделью. Это объясняется более эффективным накоплением носителей заряда в узкозонном СА-слое в ЕВFDМ-приближении относительно DDFDM-модели (рис. 5). Это происходит из-за того, что в EBFDM-приближении в области гетероперехода не накапливается такая высокая избыточная концентрация электронов из-за эффекта velocity overshoot и не происходит такого сильного искривления зоны проводимости (см. вставку на рис. 5). В области гетероперехода CA/EFD со стороны CA-слоя, где концентрация свободных носителей заряда относительно высокая, температура электронов крайне низка и близка к $300 \mathrm{~K}$ (так как тепловыделение в расчете на единичный электрон в GaAs на несколько порядков меньше, чем в $\mathrm{AlGaAs}$, за счет разницы в концентрации и в значениях параметров материалов), из-за чего значения дрейфовой скорости в EBFDM-модели высоки и могут достигать $5 \cdot 10^{8} \mathrm{~cm} / \mathrm{c}$ (более подробно данный эффект описан в [18]). Таким образом, за счет высоких значений дрейфовой скорости в EBFDM-модели (velocity overshoot) в области гетероперехода накапливается меньшая концентрация электронов, чем в DDFDM-приближении. Различия в эффективности ограничения неравновесных носителей в СА-слое присутствуют и при больших токах, однако

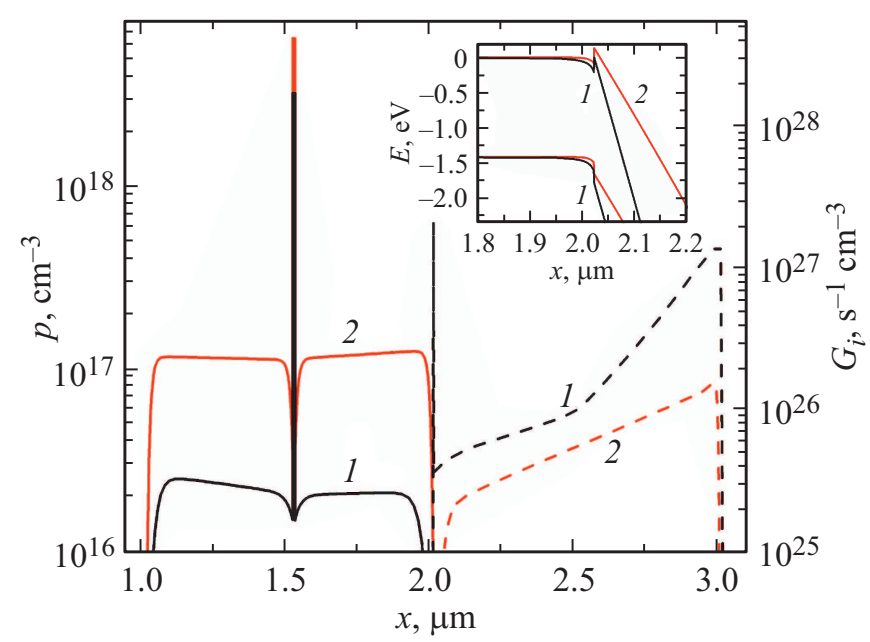

Рис. 5. Распределение концентрации электронов (сплошные линии) и скорости ударной ионизации (пунктир) в исследуемой гетероструктуре при токе $20 \mathrm{~A}$, полученных в различных моделях: 1 - DDFDM, 2 - EBFDM. На вставке показаны зонные диаграммы исследуемой гетероструктуры в области гетероперехода CA/EFD при токе $20 \mathrm{~A}$.

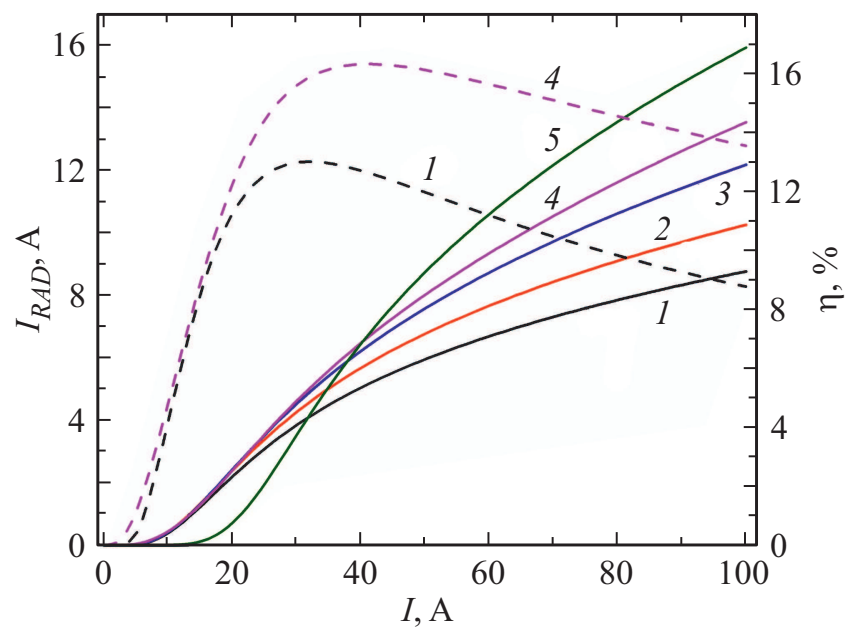

Рис. 6. Зависимости тока излучательной рекомбинации $\left(I_{R A D}\right)$ в активной области (сплошные линии) и внутренней квантовой эффективности (пунктир) от тока, протекаемого через структуру, полученные в EBFDM-модели при разных толщинах СА-слоя на основе $\mathrm{GaAs}$ (мкм): $1-1,2-0.5,3-0.2$, 4 - 0.1. Для толщины слоя GaAs в 0.1 мкм приведена также зависимость $I_{R A D}(I)$, полученная в модели DDFDM -5 . 
они нивелируются значительно более высоким темпом ударной ионизации в DDFDM-приближении.

Таким образом, так как на ток излучательной рекомбинации оказывает влияние эффективность накопления носителей заряда в узкозонном СА-слое, то мы рассмотрели влияние толщины слоя СА на ток излучательной рекомбинации в КЯ. Из рис. 6 видно, что уменьшение толщины данного слоя в 10 раз, до 0.1 мкм, увеличивает эффективность „сбора“ слоем СА дырок, сгенерированных в EFD-слое, что позволяет увеличить ток излучательной рекомбинации в КЯ более чем на $50 \%$, до $13.5 \mathrm{~A}$, а также поднять максимальную эффективность преобразования тока через структуру в ток излучательной рекомбинации в КЯ до 16\% (рис. 6).

\section{5. Заключение}

1. В работе проведено теоретическое исследование излучательной рекомбинации в изотипной гетероструктуре $n$-типа на основе материалов GaAs/AlGaAs, где неосновные носители генерируются за счет ударной ионизации. Сравнение условий транспорта, описанных в рамках моделей DD, DDFDM и EBFDM, показало, что насыщение скорости дрейфа играет существенную роль в работе прибора. Описание, полученное в рамках EBFDM-модели, показало, что имеет место эффективное накопление неравновесных носителей в узкозонном CA-слое, связанное с эффектом „velocity overshoot“" на гетерогранице CA/EFD. Именно данный эффект приводит к тому, что не происходит накопления высоких концентраций электронов на данной гетерогранице (в отличие от DDFD-модели), не происходит искривления зоны проводимости и снижения потенциального барьера. В результате эффективная излучательная рекомбинация в КЯ наблюдается уже при токах < 40 А. Для получения более точных результатов необходимы дальнейшие экспериментальные исследования.

Продемонстрирована принципиальная возможность получения излучательной рекомбинации в структурах данного типа для токов накачки 8-100 А. Показана возможность накопления электронов и дырок до концентраций $3 \cdot 10^{18} \mathrm{~cm}^{-3}$ и более при значениях тока от $10 \mathrm{~A}$, что позволяет говорить о возможности реализации условий для получения оптического усиления и стимулированной рекомбинации в изотипных гетероструктурах. Показано, что уменьшение толщины узкозонного слоя GaAs позволяет увеличить ток излучательной рекомбинации до $13.5 \mathrm{~A}$ при токе накачки $100 \mathrm{~A}$, а также увеличить максимальную внутреннюю квантовую эффективность до $16 \%$.

\section{Финансирование работы}

Авторы благодарят за финансовую поддержку при проведении исследований Российский фонд фундаментальных исследований (проект № 18-08-01130 А).

\section{Конфликт интересов}

Авторы заявляют, что у них нет конфликта интересов.

\section{Список литературы}

[1] G.W. Neudeck. Electron. Lett., 11 (17), 397 (1975).

[2] K. Hane, T. Suzuki. Jpn. J. Appl. Phys., 14 (12), 1961 (1975).

[3] J. Kostamovaara, S. Vainshtein. Breakdown phenomena in semiconductors and semiconductor devices (World Scientific, 2005) v. 36.

[4] S.O. Slipchenko, A.A. Podoskin, O.S. Soboleva, N.A. Pikhtin, T.A. Bagaev, M.A. Ladugin, A.A. Marmalyuk, V.A. Simakov, I.S. Tarasov. J. Appl. Phys., 121 (5), 054502 (2017).

[5] S.O. Slipchenko, A.A. Podoskin, O.S. Soboleva, V.S. Yuferev, V.S. Golovin, P.S. Gavrina, D.N. Romanovich, I.V. Miroshnikov, N.A. Pikhtin. Semiconductors, 53 (6), 806 (2019).

[6] L. Mollard, G. Destefanis, G. Bourgeois, A. Ferron, N. Baier, O. Gravrand, J.P. Barnes, A.M. Papon, F. Milesi, A. Kerlain, L. Rubaldo. J. Electron. Mater., 40 (8), 1830 (2011).

[7] I.I. Izhnin, K. Mynbaev, A. Voitsekhovskii, S.N. Nesmelov, S.M. Dzyadukh, A. Korotaev, V.S. Varavin, A. Korotaev, V.S. Varavin, S.A. Dvoretsky, D. Marin, M.V. Yakushev, Z. Swiatek. Semicond. Sci. Techn., 35 (11), 115019 (2020).

[8] Y. Taniyasu, M. Kasu, T. iMakimoto. Nature, 441 (7091), 325 (2006).

[9] M.L. Nakarmi, N. Nepal, C. Ugolini, T.M. Altahtamouni, J.Y. Lin, H.X. Jiang. Appl. Phys. Lett., 89 (19), 152120 (2006).

[10] O.S. Soboleva, V.V. Zolotarev, V.S. Golovin, S.O. Slipchenko, N.A. Pikhtin. IEEE T. Electron Dev., 67 (11), 4977 (2020).

[11] X. Wang, P. Crump, H. Wenzel, A. Liero, T. Hoffmann, A. Pietrzak, C.M. Schultz, A. Klehr, A. Ginolas, S. Einfeldt, F. Bugge, G. Erbert, G. Trankle. IEEE J. Quant. Electron., 46 (5), 658 (2010).

[12] D.A. Veselov, V.A. Kapitonov, N.A. Pikhtin, A.V. Lyutetskiy, D.N. Nikolaev, S.O. Slipchenko, Z.N. Sokolova, V.V. Shamakhov, I.S. Shashkin, I.S. Tarasov. Quant. Electron., 44 (11), 993 (2014).

[13] S.O. Slipchenko, A.A. Podoskin, A.V. Rozhkov, N.A. Pikhtin, I.S. Tarasov, T.A. Bagaev, M.A. Ladugin, A.A. Marmalyuk, A.A. Padalitsa, V.A. Simakov. IEEE Phot. Techn. Lett., 27 (3), 307 (2015).

[14] V.S. Yuferev, A.A. Podoskin, O.S. Soboleva, N.A. Pikhtin, I.S. Tarasov, S.O. Slipchenko. IEEE Trans. Electron Dev., 62 (12), 4091 (2015).

[15] S.N. Vainshtein, V.S. Yuferev, J.T. Kostamovaara. Solid-State Electron., 47 (8), 1255 (2003).

[16] S.N. Vainshtein, G. Duan, V.S. Yuferev, V.E. Zemlyakov, V.I. Egorkin, N.A. Kalyuzhnyy, N.A. Maleev, A.Yu. Egorov, J.T. Kostamovaara. Appl. Phys. Lett., 115 (12), 123501 (2019).

[17] S. Selberherr. Analysis and Simulation of Semiconductor Devices (Wien, N. Y., Springer Verlag, 1984).

[18] O.S. Soboleva, V.S. Yuferev, A.A. Podoskin, N.A. Pikhtin, V.V. Zolotarev, V.S. Golovin, S.O. Slipchenko. IEEE Trans. Electron Dev., 67 (2), 438 (2020).

[19] T. Grasser, T.W. Tang, H. Kosina, S. Selberherr. Proc. IEEE, 91 (2), 251 (2003).

[20] R. Stratton. Phys. Rev., 126 (6), 2002 (1962). 
[21] D.M. Caughey, R.E. Thomas. Proc. IEEE, 55 (12), 2192 (1967).

[22] B. Gonzalez, V. Palankovski, H. Kosina, A. Hernandez, S. Selberherr. Solid-State Electron., 43, 1791 (1999).

[23] Y. Apanovich, E. Lyumkis, B. Polsky, A. Shur, P. Blakey. IEEE Trans. Comput. Aid. Desing, 13 (6), 702 (1994).

[24] V. Palankovski, S. Vainshtein, V. Yuferev, J. Kostamovaara, V. Egorkin. Appl. Phys. Lett., 106 (18), 183505 (2015).

[25] S.O. Slipchenko, A.A. Podoskin, O.S. Soboleva, V.S. Yuferev, V.S. Golovin, P.S. Gavrina, D.N. Romanovich, I.V. Miroshnikov, N.A. Pikhtin. Semiconductors, 54 (5), .529 (2020).

Редактор Г.А. Оганесян

\title{
Unipolar $n$-AIGaAs/n-GaAs \\ heterostructures optimized for efficient interband radiative recombination undercurrent pumping
}

\author{
O.S. Soboleva, S.O. Slipchenko, N.A. Pikhtin \\ loffe Institute, \\ 194021 St. Petersburg, Russia
}

\begin{abstract}
An analysis of the carrier transport of an isotype $\mathrm{AlGaAs} / \mathrm{GaAs}$ heterostructure, optimized for efficient radiative recombination, under current pumping is carried out. In order to analyze the features of the carriertransport, the following models were used: drift-diffusion, drift-diffusion with a field dependent mobility, as well as the energy balance with a field dependent mobility. It is shown that the radiative recombination level in the active region at low currents is higher in the energy balance model due to the more efficient accumulation of generated by impact ionization carriers. This is explained by the presence of the velocity overshoot effect at the heterointerface, which arises due to a sharp change in the electron concentration and the electric field at the hetero junction, whereas in the drift-diffusion approximation, the drift velocity in this region is equal to the saturated one, and a there is a decrease in the potential barrier due to the accumulation of a large concentration of electrons near the heterojunction. At currents above $40 \mathrm{~A}$, the radiative recombination level in the active region is higher in the driftdiffusion approximation, which is explained by the stronger electric field and higher impact ionization rate in the layer with the electric field domain. It was demonstrated, that an increase in the radiative recombination current in the active region by more than $50 \%$ to $13.5 \mathrm{~A}$ (at a pump current of $100 \mathrm{~A}$ ) and a maximum internal quantum efficiency to $16 \%$ (at a current of $40 \mathrm{~A}$ ) with a decrease in the thickness of the carrier accumulation layer to $100 \mathrm{~nm}$.
\end{abstract}

